Dept. for Speech, Music and Hearing Quarterly Progress and Status Report

\title{
Effect on LTAS of vocal loudness variation
}

Nordenberg, M. and Sundberg, J.

journal: TMH-QPSR

volume: 45

number: 1

year: 2003

pages: $093-100$ 



\title{
Effect on LTAS of vocal loudness variation
}

\author{
Maria Nordenberg* and Johan Sundberg* \\ *KTH Voice Research Centre, Department of Speech Music and Hearing, KTH \\ Corresponding author: \\ Johan Sundberg, KTH Voice Research Centre, Department of Speech Music and Hearing, \\ SE-100 44 Stockholm, Phn +468 790 7873, Fax +468 790 7854, email pjohan@,speech.kth.se
}

\begin{abstract}
Long-term-average spectrum (LTAS) is an efficient method for voice analysis, revealing both voice source and formant characteristics. However, the LTAS contour is non-uniformly affected by vocal loudness. This variation was analysed in 15 male and 16 female untrained voices reading a text 7 times at different degrees of vocal loudness, $62 \leq$ mean Leq $\leq 91$ dB@0.3m. In all frequency bands up to $4 \mathrm{kHz}$ spectrum level was strongly and linearly correlated with overall equivalent sound level (Leq). The gain factor, i.e., the rate of level increase, varied with frequency, from about 0.5 at low frequencies to about 1.5 between 1.5 and 3 $\mathrm{kHz}$. Using the gain factors for a voice, LTAS contours could be predicted at any Leq within the measured range, with an average accuracy of 2 to $3 \mathrm{~dB}$ below 4 $\mathrm{kHz}$. Mean LTAS calculated for an Leq of $70 \mathrm{~dB}$ for each subject showed considerable individual variation for both males and females. On the other hand, the results also indicate that meaningful comparisons of LTAS, recorded e.g., before and after voice therapy, can be made, provided that the documentation includes a minimum of three recordings at different loudness levels from one recording session.
\end{abstract}

\section{Introduction}

A long-term-average spectrum, LTAS displays the average sound level in different frequency bands, and reflects both glottal and vocal tract characteristics. For example, it reflects quite clearly quasi-constant characteristics such as a singer's formant or a speaker's formant (Leino, 1994). It is frequently applied to running speech, (see e.g., Löfqvist, 1986; Wedin et al., 1978; White, 1998). It typically stabilizes after about $40 \mathrm{~s}$ (Fritzell et al., 1974). Thus, it is rather insensitive to the exact linguistic content of the speech material, but yet the use of a standard text is sometimes recommended (Löfqvist \& Mandersson, 1987).

Several methods have been tested for quantifying the information contained in an LTAS. Energy ratios or level differences between two or three frequency bands have been measured (Löfqvist \& Mandersson, 1987; Gauffin \& Sundberg, 1977; Kitzing, 1986). Hammarberg et al. (1980) found a good correlation between various perceptual characteristics of dysphonic voices and a set of level differences between different frequency bands.
The occurrence of LTAS peaks has been found to be related to voice quality (Leino, 1994; Nawka et al., 1997) or gender (Mendoza et al., 1996; White, 2001).

A complication in using LTAS for voice analysis is, however, that it is affected by vocal loudness. A typical example is shown in Fig. 1. Increased vocal loudness causes a larger increase of the LTAS level at $3 \mathrm{kHz}$ than at 0.5 $\mathrm{kHz}$. In other words, the increase of LTAS level is not uniform along the frequency scale. This causes complications in comparing LTAS curves of a voice recorded on different occasions, such as before and after therapy. For obtaining comparable LTAS data before and after therapy, Kitzing (1986) recommended that patients should read at the same degree of vocal loudness. Kitzing \& Åkerlund (1993) pointed out the need for an investigation of the effect of vocal loudness on LTAS curves.

Some data on the effect on spectrum level of vocal loudness variation have been published. For vowel sounds produced by baritone singers, the level growth in spectrum bands was 
observed to be a linear function of the $\log$ of subglottal pressure (White \& Sundberg, 2000). They found that a $10 \mathrm{~dB}$ increase of sound level produced an increase of between 15 and $20 \mathrm{~dB}$ of the partials near $2.5 \mathrm{kHz}$.

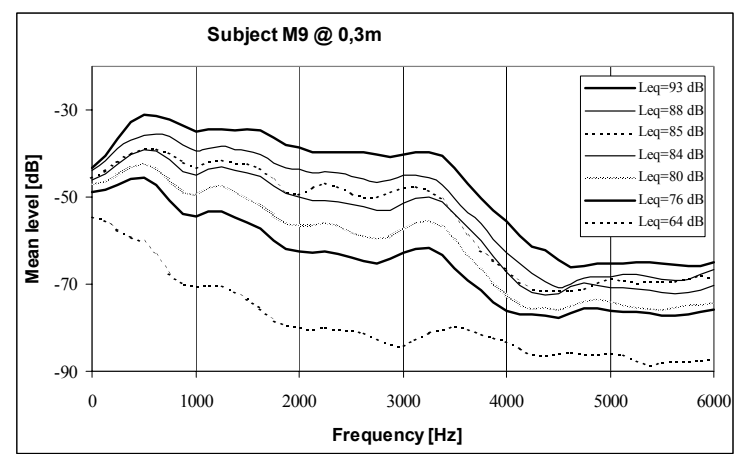

Figure 1. LTAS curves obtained from male speaker 9 for reading the same text at the indicated Leq values.

Given these findings on vowel spectra, it does not seem unlikely that similar relationships might apply also to LTAS data. Analyzing recordings of choirs that sang the same piece at three dynamic levels, Ternström (1993) found that the LTAS level in the various frequency bands was linearly related to the overall equivalent sound level, henceforth Leq. Still, the exact relationship between Leq and the levels in different frequency bands of an LTAS of running speech it as yet not known. The aim of the present investigation therefore was to analyze the relationship between Leq and LTAS levels in different frequency bands for speech produced by untrained voices at different degrees of vocal loudness.

\section{Method}

A total of 31 subjects, 16 females and 15 males, mean age 28 years (extremes 23 and 35) and 29 years (extremes 21 and 40), respectively, participated as subjects. None of them reported any voice problems or upper airway infections and none were paid for their participation. All subjects but one of the females had untrained voices.

The subjects repeatedly read a text at different degrees of vocal loudness. Before the recording begun they were allowed to acquaint themselves with the text. The reading at each degree of vocal loudness lasted for at least $40 \mathrm{~s}$, which is sufficient to produce a stable LTAS of speech (Fritzell et al, 1974). To induce different degrees of vocal loudness, a party noise was presented over ear phones (AKG MONITOR, K240) prerecorded at different levels on a DAT recorder according to a fixed scheme (Table 1). The subjects were instructed to make their voice heard over this noise. The loudest noise corresponded to an Leq of about $95 \mathrm{~dB}$. In addition, one recording was made without earphones, and in this case the subjects were instructed to read as softly as possible without whispering.

Table 1. Order and levels of the party noise presented over earphones to the subjects during the recording.

\begin{tabular}{|c|c|}
\hline $\begin{array}{c}\text { Reading } \\
\#\end{array}$ & $\begin{array}{c}\text { Level of party } \\
\text { noise [dB] }\end{array}$ \\
\hline 1 & -10 \\
\hline 2 & 0 \\
\hline 3 & -10 \\
\hline 4 & -14 \\
\hline 5 & -7 \\
\hline 6 & -18 \\
\hline 7 & No noise \\
\hline
\end{tabular}

The recordings were made in a sound treated booth, about $4 \times 3 \times 2 \mathrm{~m}$ with the subjects seated. The audio signal was picked up by a headmounted microphone (AV-JEFE, TCM 110) adjusted to a constant distance of $0.3 \mathrm{~m}$ from the mouth and recorded on DAT. To expand the dynamic range, the microphone signal was recorded on two channels with microphone gains differing by about $20 \mathrm{~dB}$.

For each subject, the microphone was calibrated by holding it next to a sound level meter (B\&K, type 2215, precision sound level meter) and recording, on both channels, a sustained vowel sound produced at two levels. The sound pressure levels observed on the sound level meter were announced on the tape. The same microphone gain was then used for the rest of the recording.

Using the Soundswell Signal Workstation 4.0 (HiTech, DSP, 1996) the recordings were digitized and transferred to sound files (sampling frequency $16 \mathrm{kHz}$ ), one for each subject. In few cases, the recording of the loudest condition contained occasional instances of overloading of some $200 \mathrm{~ms}$ duration. These 
instances were eliminated from the files, resulting in a shortening of the recording of less than $2 \%$.

LTAS were obtained from the Spectrum Section option of the Soundswell workstation, using an analysis bandwidth of $400 \mathrm{~Hz}$ and a 0-6 $\mathrm{kHz}$ frequency range. The Leq for each reading was determined by means of the Histogram option of the Soundswell workstation.

\section{Results}

In the recordings, a microphone distance of 0.3 $\mathrm{m}$ was used, and at the same time, party noise was presented over earphones, as explained above. This means that the microphone picked up the party noise which might have contributed to the LTAS. The risk for this would be greatest for the softest reading. To estimate the impact of this effect, a recording was made of the leakage for the loudest noise while the subject remained silent. The LTAS of this recording was compared with an LTAS of a randomly chosen example of the softest reading (Figure 2). The minimum difference between the two curves, occurring near $3.5 \mathrm{kHz}$, amounted to $8 \mathrm{~dB}$. Taking into account that the loudest noise was associated with much louder readings, this indicates that the noise leakage was negligible.

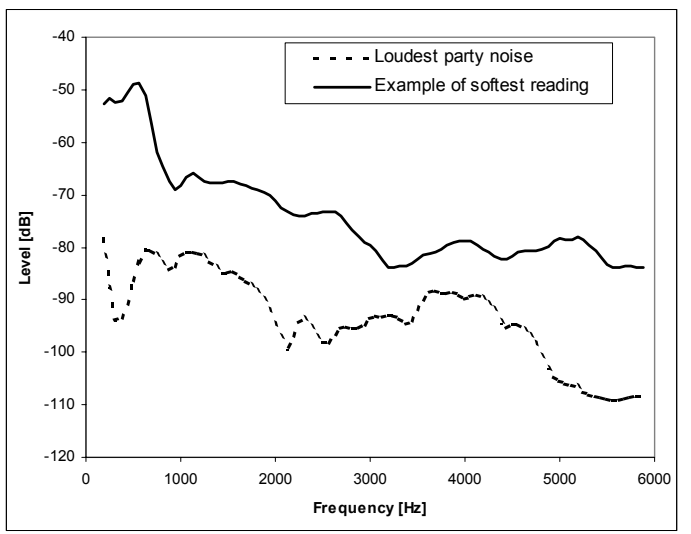

Figure 2. Solid curve: LTAS of softest reading produced by a female subject. Dashed curve: LTAS of sound leakage from the headphones to the head-mounted microphone reproducing the loudest party noise used in the experiment. During this recording of this LTAS, the subject remained silent.

Also the effect of the elimination of overloaded vowels was analyzed. Figure 3 shows examples of LTAS curves obtained from a recording before and after such elimination.
The differences are vanishingly small indicating that also in this case, the effect was negligible.

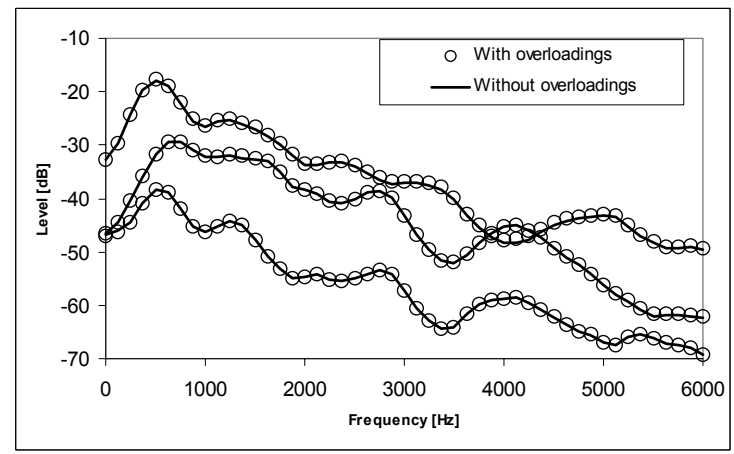

Figure 3. Comparison of LTAS obtained with (solid curve) and without (open circles) elimination of overloaded vowels.

In the analysis also unvoiced speech sounds were included. Such sounds affect LTAS curves only above about $5 \mathrm{kHz}$ (Löfqvist \& Mandersson, 1987), as can be seen also in Figure 4; the LTAS of the same recording with and without elimination of unvoiced segments are practically identical up to about $4 \mathrm{kHz}$, while a clear difference can be seen above $5 \mathrm{kHz}$. As the effect of vocal loudness variation on voice was our primary interest, we restricted the analysis of LTAS curves to the frequency range up to $4 \mathrm{kHz}$.

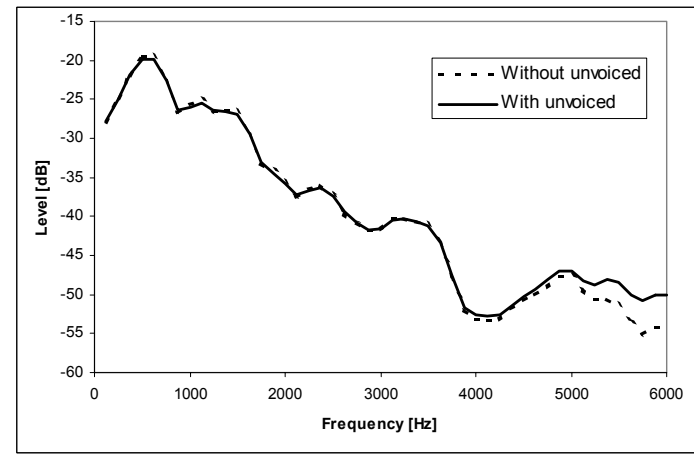

Figure 4. Comparison of LTAS obtained with (solid curve) and without (dashed curve) unvoiced segments.

To analyze the influence on LTAS curves of vocal loudness variation, the LTAS levels in each of the frequency bands were plotted as function of the associated Leq values. Figure 5 shows some typical results in the frequency bands $0.5,1.5$, and $3 \mathrm{kHz}$ for two female and two male voices. The graphs show that the LTAS levels at these frequencies increased 
linearly as function of Leq, correlation being close to 1.0. Growth rate varied with frequency and departures from a linear relationship was often greater at extreme Leq values.

Figure 6 shows the means of slope, henceforth gain factor (Ternström, 1993) correlation and intercept for the female and male voices (left and right set of graphs). Standard deviations were generally quite small for frequencies below $4 \mathrm{kHz}$. Eliminating the trained female from the female group changed the averages negligibly. Hence, these averages can be assumed to be representative for untrained female and male voices.

The gain factor was well below 1.0 in the low frequency range, approximated 1.6 (females) and 1.4 (males) in the range $1.5-3$
$\mathrm{kHz}$ and less at higher frequencies. This implies that for the female and male subjects a $10 \mathrm{~dB}$ increase of Leq was associated with an average of 16 and $14 \mathrm{~dB}$ increase in the $1.5-3 \mathrm{kHz}$ frequency range. At about $0.5 \mathrm{kHz}$ the gain factor was close to 1.0 for both females and males, indicating that Leq can be approximated from the LTAS level at that frequency.

In the figure, it can be seen that high gain factor values were associated with low intercept values. This relationship is illustrated in Figure 7. For both females and males the curves make a turn, at the gain factor of 1.6 for the females and at 1.4 for the males, reflecting the fact that LTAS levels are lower in the high frequency end than in the range $0.5-4 \mathrm{kHz}$.
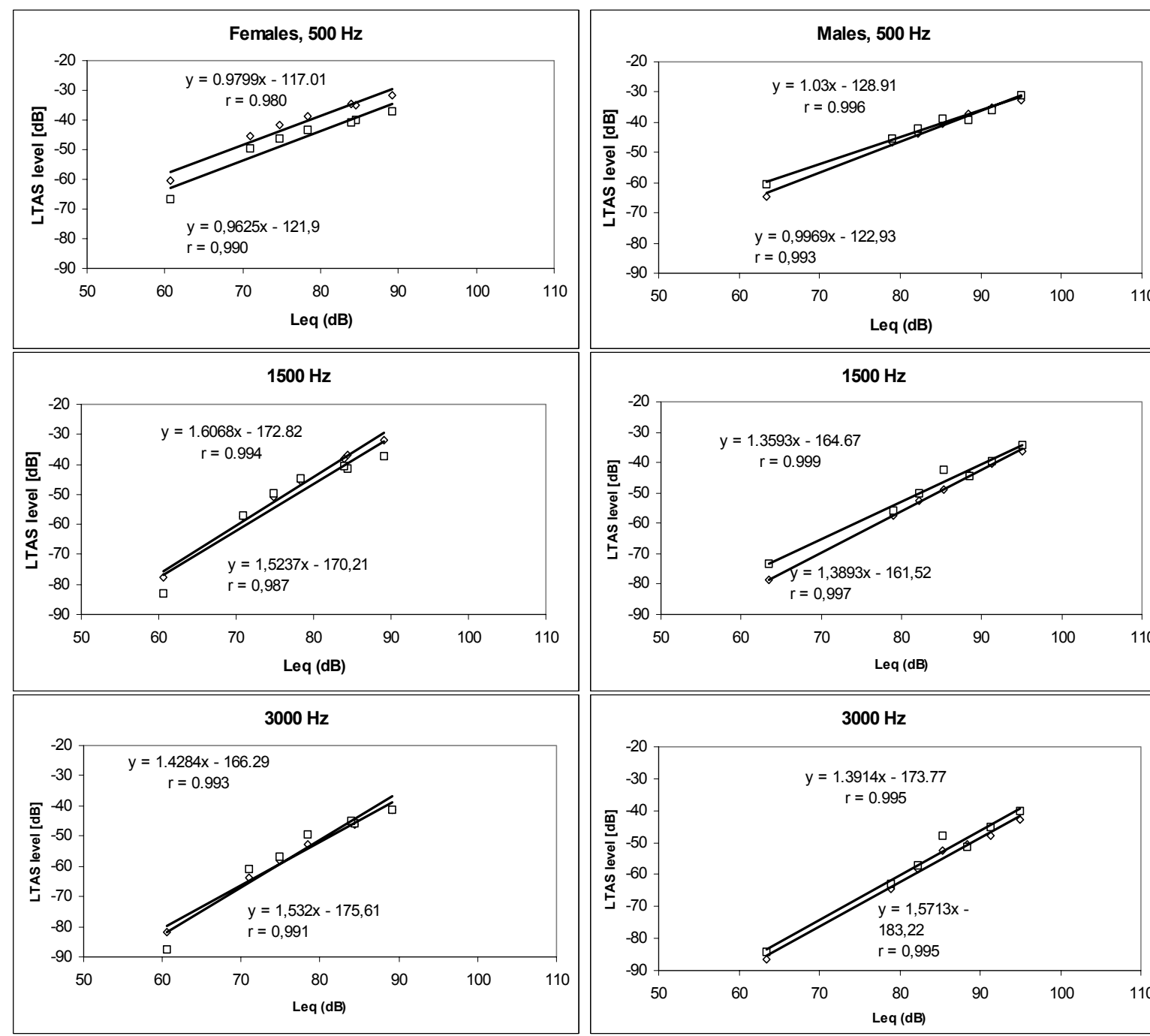

\begin{tabular}{|l|l|}
\hline \hline-20 & $1500 \mathrm{~Hz}$ \\
\hline
\end{tabular}
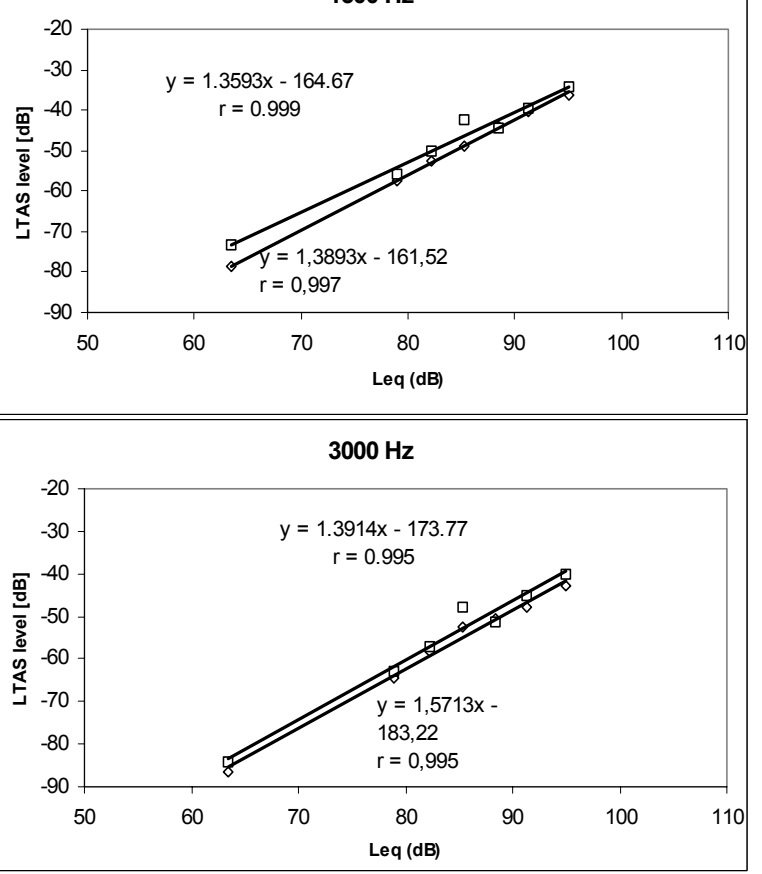

Figure 5. Relationship between LTAS levels in the indicated frequency bands for female subjects W8 and W14 (left panels, diamonds and squares, respectively) and for male subjects M2 and M9 (right panels, diamonds and squares, respectively). The lines represent the best linear fit of the data points, and the associated equations and correlations $r$ are also shown. 


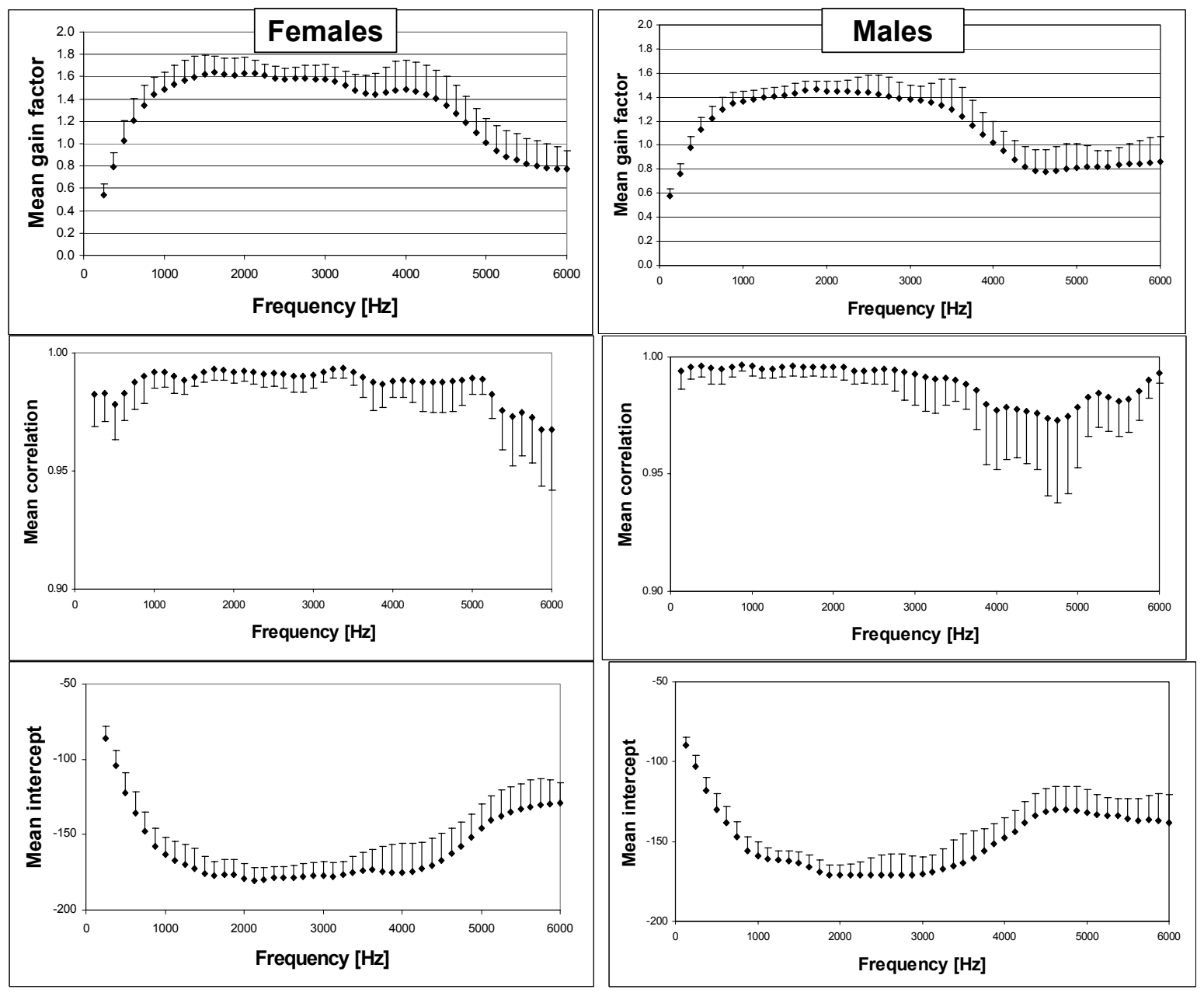

Figure 6. Gain factors (upper panel), correlations (middle panel) and intercepts (lower panel), averaged across the female and male subjects (left and right set of graphs, respectively). Bars represent 1 standard deviation.

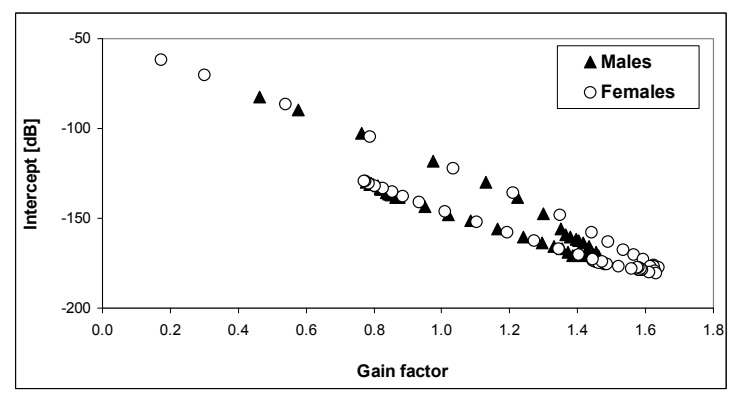

Figure 7. Relationship between mean gain factor and mean intercept for the female and male subjects.

The high correlation values suggest that for the degrees of vocal loudness analyzed here Leq can be used to predict LTAS levels in the frequency range up to about $4 \mathrm{kHz}$. The greatest departures from a linear relationship between LTAS levels and Leq were often noted for the highest and lowest Leq values. Therefore, an attempt was made to predict LTAS curves at extreme Leq values for all voices, and the absolute value of the observed - predicted difference was computed and averaged across subjects. Figure 8 shows the results. The mean error was close to 2 and $1 \mathrm{~dB}$ for the female and male subjects, respectively, SD being close to $1 \mathrm{~dB}$ for both groups. The error showed a trend to increase near $4 \mathrm{kHz}$, particularly for the males. Recalling that these deviations at Leq extremes should represent the worst case, the results show that LTAS curves can be predicted with a reasonable accuracy, given the Leq value and the equations relating this value to LTAS level in the various frequency bands.

An attempt was made to derive normative LTAS curves for female and male voices at an Leq of $70 \mathrm{~dB}$. Figure 9 presents these average LTAS curves. The SD values of approximately $4 \mathrm{~dB}$ reflect a rather substantial inter-individual 

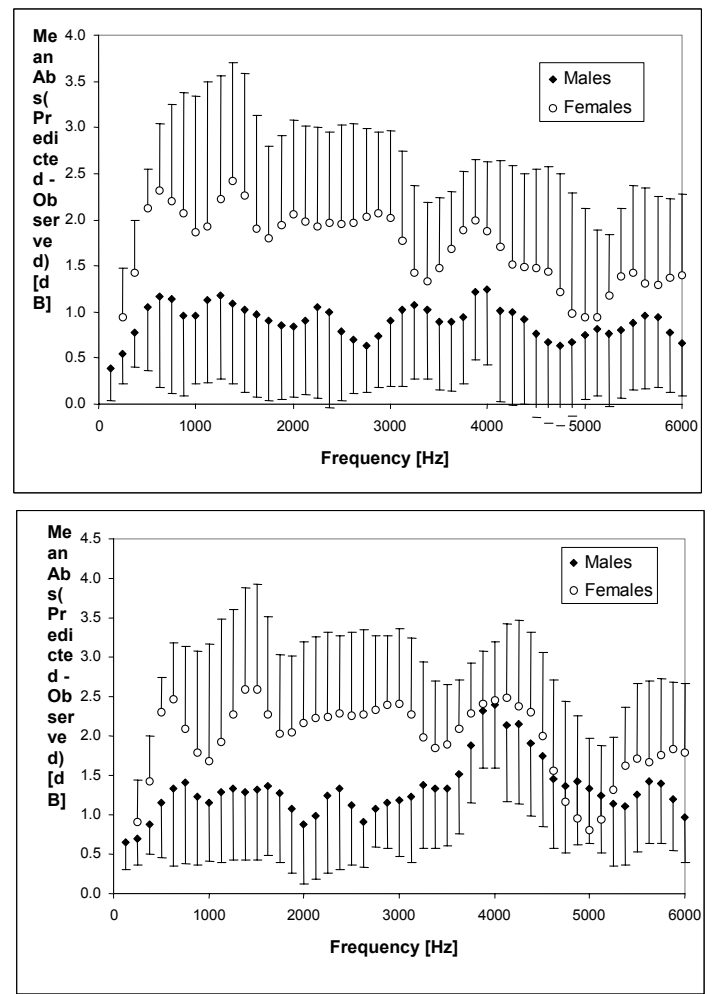

Figure 8. Results from the attempt to predict LTAS levels for each subject's softest and loudest reading (panels above and under). The graphs show the absolute difference between calculated and observed LTAS levels, averaged across female and male subjects. Bars represent 1 standard deviation.

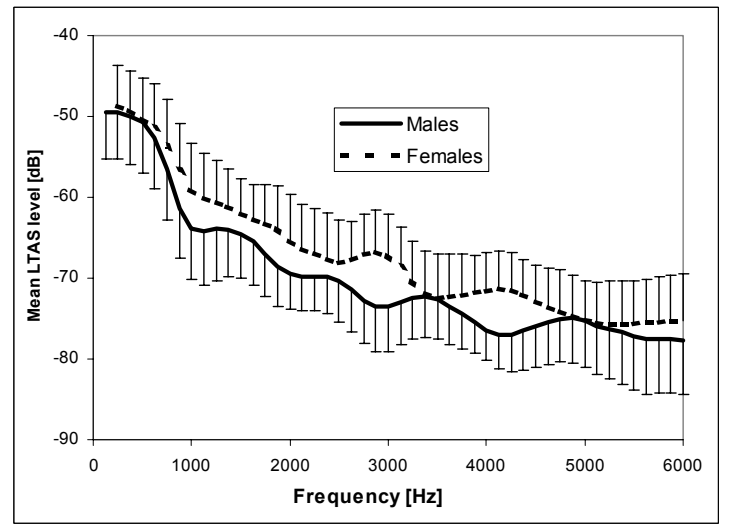

Figure 9. Calculated LTAS curves, averaged across female and male subjects, for $70 \mathrm{~dB}$ Leq. Bars represent 1 standard deviation.

variation within the two groups. In the frequency range $1-4 \mathrm{kHz}$, the LTAS curve for the females is on average $3.5 \mathrm{~dB}$ higher than that of the males. Slight peaks can be observed at 2.4 and $3.4 \mathrm{kHz}$ in the male LTAS and at 2.9 and
$4.1 \mathrm{kHz}$, i.e., at about $20 \%$ higher frequencies in the female LTAS. This is close to the $17 \%$ difference observed by Fant (Fant, 1975) for the third formant in female and male voices. The slightly higher levels between 1 and $4 \mathrm{kHz}$ in the female as compared to the male LTAS curves may suggest a higher degree of vocal effort spent by the females in order to reach an Leq of $70 \mathrm{~dB}$.

\section{Discussion and Conclusions}

The observed effects of vocal loudness variation on LTAS curves is by no means unexpected. It has been previously shown for vowel spectra (White \& Sundberg, 2000) as well as for LTAS of choral singing (Ternström, 1993) as well as for sustained vowel sounds (e.g., Bloothooft \& Plomp, 1986; Holmberg et al., 1995). The effect would originate from the voice source. An increase of sound level is produced by an increase of subglottal pressure, which, in turn, affects the waveform of the transglottal airflow. Thus, it has been shown that an increase of subglottal pressure in baritone singer voices lengthens the closed phase and increases the peak-to-peak amplitude of the glottal flow waveform. Both these effects contribute to increasing the negative peak amplitude of the differentiated flow waveform, which, in turn, determines the amplitudes of the overtones of the source spectrum (Fant et al., 1985). The amplitudes of the higher partials are particularly sensitive to the return phase, i.e., the speed at which the airflow decreases in the final part of the open phase. The substantial influence of loudness on spectrum levels limits the value of comparing acoustic data produced at different degrees of vocal loudness.

An interesting question is why the females showed higher LTAS levels between 1 and 4 $\mathrm{kHz}$ as compared to the males. This may suggest that females tend to need a higher degree of vocal effort to reach a given Leq than males. Interestingly, Gelfer and Young (1997) noted that in conversational speech females produced a sound level about $2.5 \mathrm{~dB}$ lower than that of males. According to our results a $2.5 \mathrm{~dB}$ difference in Leq would cause the LTAS level between 1 and $4 \mathrm{kHz}$ to increase by $1.6 * 2.5=4$ $\mathrm{dB}$. This value is similar to the average level difference we observed between the male and female LTAS in the $1-4 \mathrm{kHz}$ range. This may suggest that an Leq of $70 \mathrm{~dB}$ requires a higher subglottal pressure in females than in males. 
Some support for this assumption can be seen in our observation that the males' mean Leq at softest and loudest reading was $1 \mathrm{~dB}$ higher than that of the females. In any event, it would be interesting to compare subglottal pressures in untrained female and male voices phonating at identical Leq.

The dynamic range analyzed here was about $28 \mathrm{~dB}$ for both the female and the male subjects. However, the loudest noise did not cause the subjects to produce maximum loudness. Under such conditions, a different relationship between Leq and LTAS levels can be expected. Therefore, we assume that the linear relationship between Leq and LTAS level apply to a normal range loudness variation range (Ternström, 2003).

Our subjects wore headphones while reading the text, which should have produced an occlusion effect, i.e., that the subjects heard their own voices in a distorted way (Gelfand, 1990). This raises the question if our results would have been different had the subjects not worn earphones. This, however, seems unlikely. The subjects did not have earphones when producing the softest reading, and the values observed under this condition did not differ appreciably from those observed under the remaining loudness conditions. Therefore, there seems little reason to assume that the linear relationship between LTAS level and Leq were affected by the earphones.

We calculated an LTAS for an Leq of $70 \mathrm{~dB}$ for all voices and the LTAS averaged across subjects were shown in Figure 9. It should be noted, however, that the shape of an LTAS curve is strongly influenced by the analysis bandwidth. We used a $400 \mathrm{~Hz}$ bandwidth, which increased the comparability of LTAS obtained from different voices, regardless of their fundamental frequencies. However, because of the great SD it would be inappropriate to regard these LTAS as normative data.

On the other hand, our results show that the LTAS for any Leq within the normal range of loudness variation can be calculated with fair accuracy, given the relationship between Leq and LTAS levels in the various frequency bands. This relationship varies between individuals, and must therefore be determined for each individual voice. The procedure would be simply to ask the subject to read a text at three different degrees of vocal loudness. From the LTAS obtained from these readings, the individual subject's Leq - LTAS level equations can be determined. This simple procedure would allow a documentation of voice changes, e.g., after training and/or therapy, that would be far more informative than a documentation based on a comparison of LTAS produced at different Leq.

\section{Acknowledgements}

This is a condensed version of co-author MN's master's thesis at the department of Logopedics and Phoniatrics at Lund University, carried out at the department of Speech Music and Hearing, $\mathrm{KTH}$, under the supervision of co-author JS. The kind and generous assistance of Svante Granqvist at this department and the patient participation of the 31 subjects is gratefully acknowledged.

\section{References}

Bloothooft G \& Plomp R (1986). The sound level of the singer's formant in professional singing; $J$ Acoust Soc Am 79: 2028-2033.

Fant G (1975). Nonuniform vowel normalization STL-QPSR, KTH, 1975/2-3: 1-19.

Fant G, Liljencrants J \& Lin Q (1985) A fourparameter model of glottal flow, STL-QPSR, KTH 1985/4: 1-13.

Fritzell B, Hallén O \& Sundberg J (1974). Evaluation of Teflon injection procedures for paralytic dysphonia. Folia Phoniatrica, 26: 414-421.

Gauffin J \& Sundberg J (1977). Clinical application of acoustic voice analysis. Part II: Acoustic analysis, results and discussion. STL-QPSR, KTH 1977/2-3: 39-43.

Gelfand S (1990). Hearing, 2nd ed, New York: Marcel Dekker Inc.

Gelfer M \& Young S (1997). Comparison of intensity measures and their stability in male and female speakers. Journal of Voice, 11: 178-186.

Hammarberg B, Fritzell J, Gauffin J, Sundberg J \& Wedin L (1980). Perceptual and acoustic correlates of abnormal voice qualities. Acta Otolaryngologica, 90: 441-451.

Holmberg E, Hillman R, Perkell J, Guiod P, Goldman S (1995). Comparisons among aerodynamic, electroglottographic, and acoustic spectral measures of female voice, J Speech Hear Res 38: 1212-1223.

Kitzing P \& Åkerlund L (1993). Long-time average spectrograms of dysphonic voices before and after therapy. Folia Phoniatrica, 45: 53-61.

Kitzing P (1986). LTAS criteria pertinent to the measurement of voice quality. Journal of Phonetics, 14: 477-482.

Leino T (1994). Long-term average spectrum study on speaking voice quality in male actors. In: Friberg A, Iwarsson J, Jansson E \& Sundberg J, eds. SMAC 93 (Proceedings of the Stockholm Music Acoustics Conference 1993), Stockholm: Publication $\mathrm{Nr} 79$ issued by the Royal Swedish Academy of Music: 206-210. 
Löfqvist A (1986). The long-time-average spectrum as a tool in voice research. Journal of Phonetics, 14: 471-475.

Löfqvist A \& Mandersson B (1987). Long-time average spectrum of speech and voice analysis. Folia phoniatrica, 39: 221-229.

Mendoza E, Valencia N, Munoz J \& Trujillo H (1996). Differences in voice quality between men and women: Use of long-term average spectrum (LTAS).

Nawka T, Anders LC, Cebulla M, Zurakowski D (1997). The speakers formant in male voices. Journal of Voice, 11: 422-428.

Ternström S (1993) Long-time average spectrum characteristics of different choirs in different rooms. Voice (UK), 2: 55-77.

Ternström S (2003). Very loud speech over simulated environmental noise tends to have a spectral peak in the F1 region, $J$ Acoust Soc Am 113:4, part 2, 2296 (abstract).

Wedin S, Leanderson R \& Wedin L (1978). Evaluation of voice training. Spectral analysis compared with listeners' judgements. Folia phoniatrica, 30: 103-112.

White P (1998). The effect of vocal intensity variation on children's voices using long-term average spectrum (LTAS) analysis, Log Phon Vocol 23: 111-120.

White P (2001). Long-term average spectrum (LTAS) analysis of sex- and gender-related differences in children's voices. Logopedics Phonetics Vocology, 26: 97-101.

White P \& Sundberg J (2000). Spectrum effects of subglottal pressure variation in professional baritone singers, TMH-QPSR, KTH, 2000/4: 29-32. 